\title{
New DTR Estimation Method Without Measured Solar and Wind Data
}

\author{
Zhan-Feng Ying*, Yuan-Sheng Chen ${ }^{\dagger}$ and Kai Feng*
}

\begin{abstract}
Dynamic thermal rating (DTR) of overhead transmission lines can provide a significant increase in transmission capacity compared to the static thermal rating. However, the DTR are usually estimated by the traditional thermal model of overhead conductor that is highly dependent on the solar, wind speed and wind direction data. Consequently, the estimated DTR would be unreliable and the safety of transmission lines would be reduced when the solar and wind sensors are out of function. To address this issue, this study proposed a novel thermal model of overhead conductor based on the thermal-electric analogy theory and Markov chain. Using this thermal model, the random variation of conductor temperature can be simulated with any specific current level and ambient temperature, even if the solar and wind sensors are out of function or uninstalled. On this basis, an estimation method was proposed to determine the DTR in the form of probability. The laboratory experiments prove that the proposed method can estimate the DTR reliably without measured solar and wind data.
\end{abstract}

Keywords: Dynamic thermal rating, Overhead transmission lines, Conductor temperature, Markov chain

\section{Introduction}

The thermal rating of an overhead transmission line is the maximum allowable current that prevent the line overheating. Historically, the requirement for providing a reliable service has led the electricity sector to adopt static thermal ratings (STR), which have been calculated in the worst weather conditions (low wind speed, high ambient temperature and solar radiation). As a consequence, many overhead transmission lines cannot be fully utilized to transmit power [1]. With the increasing of power transmission cost, there is great pressure to improve the currentcarrying capacity using existing infrastructure as far as possible. Therefore, the dynamic thermal rating (DTR) of transmission line has been developed rapidly in recent decades [2-4].

Different form the STR, the DTR is determined with the conductor tension or conductor temperature, so that the carrying capacity can be dynamically corrected and fully exploited. Since the conductor temperature can be measured relatively easily and can reflect local hot spot of conductor, many researchers tend to obtain DTR by using conductor temperature rather than tension [5-7]. In this background, IEEE proposed a standard conductor thermal model to calculate the conductor temperature and the DTR under different weather conditions [8]. However, a great deal of uncertainty exist in the weather data measured by DTR system because of the variation of weather conditions along the line, and between the lines and the weather

$\dagger$ Corresponding Author: School of Energy and Power Engineering, NanJing University of Science and Technology, China.

(chenys@njust.edu.cn)

* School of Energy and Power Engineering, NanJing University of Science and Technology, China. (\{yingzhanfeng, Kaifeng\}@163.com) Received: August 5, 2016; Accepted: December 28, 2016 station. As a result, the DTR cannot be exactly calculated but only estimated.

A great number of studies have been carried out for enhancing reliability of DTR estimation. In [9] and [10], the DTR is forecasted by Monte Carlo simulation and statistical weather models. A fuzzy theory was applied to characterize weather uncertainty for DTR estimation in [11]. By using affine arithmetic, reference [12] determined the thermal rating taking into account the parameter uncertainty interdependencies. In [13], a nonlinear adaptive modeling technique based on Local Learning theory was used to reduce the impact of weather uncertainty on the DTR estimation. The influences of weather measured data and measurement time intervals on the performance of DTR estimation were analyzed in [14].

The above-mentioned studies were carried out on the basis of the weather sensors which have to work properly. However, because the solar and wind sensors have to be exposed in the air to ensure measurement accuracy, they would be damaged by some unpredictable factors, such as hail impacting, mechanism wear, dust deposit and atmospheric corrosion. These sensors are difficult to be maintained or calibrated timely since they were set up at a height above ground comparable to the height of transmission line [14]. After a long-term operation, the measured data from weather sensors are not fully credible [15]. Since the thermal model of conductor, which widely used at present, is highly dependent on the solar, wind speed and wind direction data, the estimated DTR would be unreliable when the solar and wind sensors are out of function. Therefore, it is very important to reduce dependence on the solar and wind sensors in the DTR estimation. In this way, the DTR estimation will be more reliable. Besides that, the DTR system structure will be 
simplified and then the cost of DTR system will be reduced.

In this study, a novel thermal model of overhead conductor is proposed with the thermal-electric analogy theory and Markov chain. Then, an estimation method is proposed to determine DTR without measured solar and wind data. In order to demonstrate the effectiveness of the proposed estimation method, an experimental platform was set up and the validation experiments are presented.

\section{Traditional Thermal Model of Overhead Conductor}

The thermal model plays a key role in the DTR estimation because it can describe the thermal behavior of overhead conductor under the different current and weather conditions. The traditional and most widely used thermal model for DTR estimating comes from IEEE standard and can be expressed as [8]

$$
C \frac{d T_{c}}{d t}=q_{J}+q_{s}-q_{c}-q_{r}
$$

where $T_{c}$ is conductor temperature $\left({ }^{\circ} \mathrm{C}\right) . C$ is thermal capacity of conductor $\left(\mathrm{J} / \mathrm{m}^{\circ} \mathrm{C}\right) . \quad q_{J}$ and $q_{s}$ are, respectively, Joule and solar heat gains $(\mathrm{W} / \mathrm{m}) . q_{c}$ and $q_{r}$ are, respectively, the convective and radiated heat losses $(\mathrm{W} / \mathrm{m})$.

The parameters in the traditional thermal model are calculated by:

$$
\begin{aligned}
q_{c}= & K_{\text {angle }}\left[1.01+0.371\left(\frac{D \rho_{f} V_{w}}{\mu_{f}}\right)^{0.52}\right] \\
& \times k_{f}\left(T_{c}-T_{a}\right) \text { (for low wind speed) } \\
q_{c}= & K_{\text {angle }} 0.1695\left(\frac{D \rho_{f} V_{w}}{\mu_{f}}\right)^{0.6} \\
& \times k_{f}\left(T_{c}-T_{a}\right)(\text { for high wind speed }) \\
q_{J}= & I^{2} R_{T c} \\
q_{s}= & \alpha K_{\text {solar }} Q_{s} \sin (\theta) A^{\prime} \\
q_{r}= & 0.0178 D \varepsilon\left[\left(\frac{T_{c}+273}{100}\right)^{4}-\left(\frac{T_{a}+273}{100}\right)^{4}\right]
\end{aligned}
$$

where $\rho_{f}$ is the air density $\left(\mathrm{kg} / \mathrm{m}^{3}\right) . \quad \mu_{f}$ is absolute air viscosity $(\mathrm{kg} / \mathrm{m} \cdot \mathrm{h}) . \quad k_{f}$ is the thermal conductivity of air that depends on wind speed $\left(\mathrm{W} / \mathrm{m}^{\circ} \mathrm{C}\right) . K_{\text {angle }}$ is wind direction factor and $V_{w}$ is wind speed $(\mathrm{m} / \mathrm{s}) . \quad I$ is current flow though conductor (A). $R_{T c}$ is AC resistance of conductor at temperature $T_{c}(\mathrm{Ohm}) . Q_{s}$ is solar radiated heat flux rate $\left(\mathrm{W} / \mathrm{m}^{2}\right) . K_{\text {solar }}$ is solar altitude correction factor. $\theta$ is effective angle of incidence of the sun's rays (degrees). $A^{\prime}$ is projected area of conductor per unit length $\left(\mathrm{m}^{2} / \mathrm{m}\right) . \quad D$ is conductor diameter $(\mathrm{m}) . \quad \alpha$ and $\varepsilon$ are, respectively, solar absorptivity and emissivity with the range from 0.23 to 0.91 .

The traditional thermal model provides a simple method to estimate maximum allowable current by assumption that the conductor temperature has been closed to maximum allowable value and the derivative $d T_{c} / d t$ is set to zero, as shown in Eq.(7).

$$
I_{\max }=\left(\frac{q_{c}+q_{r}-q_{s}}{R_{T c}}\right)^{\frac{1}{2}}
$$

where $I_{\max }$ is the maximum allowable current.

Since the line DTR is defined as the maximum allowable current varying with the real-time weather conditions, it can be estimated dynamically by using Eq. (7) at any time instant. However, the traditional thermal model is highly dependent on the weather conditions, and the estimated DTR is probably unreliable when the weather sensors are out of function. To reduce the dependence on weather data in DTR estimation, a novel thermal model of overhead conductor is proposed with the thermal-electric analogy theory and Markov chain in the next section.

\section{Novel Thermal Model of Overhead Conductor}

\subsection{Model principle}

According to the thermal-electric analogy theory [16,17], an analogous relationship exists between the Fourier theory and Ohm's law. That means the convective heat loss can be calculated as

$$
q_{c}=\frac{T_{c}-T_{a}}{R_{e}}
$$

wher e $R_{e}$ is the equivalent convective thermal resistance $\left({ }^{\circ} \mathrm{C} / \mathrm{W}\right)$ and it represents cooling effect of wind on the conductor.

Because conductors operate over a relatively limited temperature range, the study in [18] indicated that linearization of radiated heat losses can be achieved with an acceptably small error in the conductor temperature calculation. In another word, the parameter $q_{r}$, which essentially depends on the biquadrate of conductor temperature and ambient temperature, can also be calculated on the basis of Ohm's law

$$
q_{r}=\frac{T_{c}-T_{a}}{R_{r}}
$$

where $R_{r}$ is the equivalent thermal resistance of radiation 
$\left({ }^{\circ} \mathrm{C} / \mathrm{W}\right)$. Considering that the conductor temperature is always higher than ambient temperature during the power transmission, $R_{r}$ represents cooling effect of radiation on the conductor.

By substituting Eq. (8) and Eq. (9) into Eq. (1), the traditional thermal model can be rewritten as a first-order linear equation

$$
C \frac{d T_{c}}{d t}=q_{J}+q_{s}-\frac{T_{c}-T_{a}}{R_{x}}
$$

where $R_{x}=R_{e} R_{r} /\left(R_{e}+R_{r}\right)$ is the integrated equivalent thermal resistance (IETR, ${ }^{\circ} \mathrm{C} / \mathrm{W}$ ) and it represents total heat dissipation capacity of conductor.

According to linear system theory, the conductor temperature is equivalent to the system response. Thus, after using backward difference method to discretize Eq. (10), the conductor temperature at the time $n$ and $n-1$ can be expressed as

$$
\begin{gathered}
T_{c}^{n}=T_{c}^{n-1}-\frac{\Delta t}{C}\left(q_{J}^{n}+q_{s}^{n}-\frac{T_{c}^{n}-T_{a}^{n}}{R_{x}^{n}}\right) \\
T_{c}^{n-1}=T_{c}^{n-2}-\frac{\Delta t}{C}\left(q_{J}^{n-1}+q_{s}^{n-1}-\frac{T_{c}^{n-1}-T_{a}^{n-1}}{R_{x}^{n-1}}\right)
\end{gathered}
$$

where $\Delta t$ is the discrete time step size.

Among the weather conditions, the wind speed and wind direction are recognized as the most variant parameters, because their magnitudes can be changed in tens of seconds. The solar radiation is mainly affected by the sun angle and the cloud thickness, both of which change slowly relative to the wind condition. Therefore the variation of solar heat gains $q_{s}$ are relatively small during such time scale. That means the solar heat gains can be assumed to be nearly unchanged if $\Delta t$ is set to tens of seconds.

Therefore, by subtracting Eq. (12) from Eq. (11), we have

$$
T_{c}^{n}-2 T_{c}^{n-1}+T_{c}^{n-2}=\frac{\Delta t}{C}\left(q_{J}^{n-1}-q_{J}^{n}+\frac{T_{c}^{n}-T_{a}^{n}}{R_{x}^{n}}-\frac{T_{c}^{n-1}-T_{a}^{n-1}}{R_{x}^{n-1}}\right)
$$

Considering that the conductor electrical resistance is commonly expressed as a linear function of conductor temperature [19], the Joule heat gains $q_{J}^{n}$ can be calculated as

$$
q_{J}^{n}=\left(I^{n}\right)^{2} R_{r e f}\left[1+\alpha_{r e f}\left(T_{c}^{n}-T_{r e f}\right)\right]
$$

where $T_{\text {ref }}$ is the reference temperature of conductor $\left({ }^{\circ} \mathrm{C}\right)$, $R_{r e f}$ is the conductor electrical resistance at temperature $T_{r e f}(\mathrm{Ohm})$, and $\alpha_{\text {ref }}$ is thermal resistivity coefficient of conductor $\left(\mathrm{Ohm} /{ }^{\circ} \mathrm{C}\right)$.

By substituting Eq. (14) into Eq. (13), a novel thermal model of conductor can be proposed as

$$
T_{c}^{n}=\frac{2 T_{c}^{n-1}-T_{c}^{n-2}+Y^{n}-Z^{n}}{X^{n}}
$$

where

$$
\begin{gathered}
A^{n}=1+\frac{\Delta t}{C}\left[\left(I^{n}\right)^{2} R_{r e f} \alpha_{r e f}-\frac{1}{R_{x}^{n}}\right] \\
Y^{n}=\frac{\Delta t}{C}\left[q_{J}^{n-1}-\left(I^{n}\right)^{2} R_{r e f}\left(1-\alpha_{r e f} T_{r e f}\right)\right] \\
Z^{n}=\frac{\Delta t}{C}\left[\frac{T_{a}^{n}}{R_{x}^{n}}+\frac{T_{c}^{n-1}-T_{a}^{n-1}}{R_{x}^{n-1}}\right]
\end{gathered}
$$

Eq. (15) indicates that the conductor temperature in any specific current level and ambient temperature can be simulated by the proposed model if the value of IETR are obtained at different time instant. In this case, the solar and wind data are no longer as the essential inputs for describing the thermal behavior of overhead conductor. This allows DTR to be estimated even the solar and wind sensors do not work.

However, due to the fluctuating wind conditions, the IETR is essentially a random process. In order to further improve the reliability of DTR estimation, the conductor temperature should be simulated with random changes caused by the random IETR. That means the IETR was required to be simulated as a random process before the proposed model is applied into DTR estimation.

\subsection{Random simulation of IETR}

Random simulation of IETR involves two issues, one is how to obtain historical IETR and the other is how to generate time series of simulated IETR.

In practical engineering, the IETR is difficult to be measured because the apparatus for thermal resistance measurement is too large and heavy to be fixed on the conductor. Fortunately, most of DTR systems measure the conductor temperature directly, or obtain it indirectly from the conductor tension or the sag [20-22]. Therefore by substituting historical measurements that include conductor temperature into Eq. (13), historical IETR $R_{x}^{n}$ can be calculated as

$$
R_{x}^{n}=\frac{T_{c}^{n}-T_{a}^{n}}{F^{n}+H^{n}+q_{J}^{n}-q_{J}^{n-1}}
$$

where

$$
\begin{gathered}
F^{n}=\frac{C}{\Delta t}\left(T_{c}^{n}-2 T_{c}^{n-1}+T_{c}^{n-2}\right) \\
H^{n}=\frac{T_{c}^{n-1}-T_{a}^{n-1}}{R_{x}^{n-1}}
\end{gathered}
$$


Eq. (19) to Eq. (21) show that an initial value of IETR is essential required for calculating historical IETR. This initial value can be obtained from Eq. (11) when the solar heat gains $q_{s}$ is zero (in night or in cloudy day), since the IETR is the only one unknown parameter exists in Eq. (11) at this time.

After obtaining the historical IETR, the time series of simulated IETR can be generated if a suitable simulation method are employed. Many works have proved that the Markov chain is an effective theory to simulate the random variation of weather conditions [23-26]. Because the randomness of IETR is mainly caused by the wind fluctuation, the Markov chain is employed to obtain the time series of simulated IETR in this study.

The Markov chain is a discrete random process that assumes the future states of random variables only depends on the current states. It can be used to generate a series of random variables from the initial states by the conditional transition probability. Suppose the state of historical IETR at the time instant $n-1$ is denoted by $i$ and the state at the time instant $n$ is denoted by $j$, the conditional transition probability of IETR from state $i$ to state $j$ in the Markov chain can be expressed as

$$
p_{i j}=P\left(R_{x}^{n}=j \mid R_{x}^{n-1}=i\right)
$$

where $p_{i j}$ is the conditional probability.

Suppose the state number of historical $R_{x}^{n}$ is finite and denoted by $L$, the Markov chain can be expressed as a state transition probability matrix $\mathbf{P}$

$$
\mathbf{P}=\left(\begin{array}{cccc}
p_{11} & p_{12} & \cdots & p_{1 L} \\
p_{21} & p_{22} & \cdots & p_{2 L} \\
\vdots & \vdots & \ddots & \vdots \\
p_{L 1} & p_{L 2} & \cdots & p_{L L}
\end{array}\right)
$$

Each row in the transition matrix is a conditional probability and the sum of each row is one.

After the elements of matrix $\mathbf{P}$ is calculated from the historical IETR, the time series of simulated IETR can be generated by elements of transition matrix with a random initial state.

\section{Proposed DTR Estimation Method}

Based on the proposed thermal model and random simulation of IETR, an estimation method was developed to determine the DTR in the form of probability. This method is implemented according to the following steps:

Step 1): Acquire the time series $\left\{I^{n}\right\},\left\{T_{e}^{n}\right\}$ and $\left\{T_{c}^{n}\right\}$, $n \in[1, N]$, by the DTR system. These series are composed by historical measurements and $N$ is the length of series.
Step 2): Calculate historical IETR with the historical measurements by Eq. (19).

Step 3): Divide historical IETR into $L$ states and construct Markov chain of historical IETR.

Step 4): Generate time series of simulated IETR $\left\{\hat{R}_{x}^{m}\right\}$, $m \in[1, M]$, based on the Markov chain, where $M$ is the length of time series $\left\{\hat{R}_{x}^{m}\right\}$.

Step 5): Generate time series of simulated conductor temperature $\left\{\hat{T}_{c}^{m}\right\}$ using Eq. (15) with $\left\{\hat{R}_{x}^{m}\right\}$, the different current level and the present ambient temperature.

Step 6): Set a maximum allowable conductor temperature $T_{c \max }$ and calculate conductor overheating probability based on the series $\left\{\hat{T}_{c}^{m}\right\}$.

Step 7): Determine DTR at the present weather condition by searching a maximum current level whose corresponding overheating probability is less than a preset maximum overheating probability $P_{\max }$.

Obviously, with the advantage of the proposed thermal model, this method is able to estimate DTR without measuring the solar and wind data.

According to the statistics, the larger $N$ and $L$ in the proposed estimation method are selected, the more accurate Markov chain of IETR will be constructed, but it comes with the increasing of computation load. Therefore the value of $N$ and $L$ should be selected depend on the computing power of DTR system. The value of $M$ determines the amount of simulated conductor temperature. To improve calculating accuracy of conductor overheating probability, the value of $M$ should also be as large as possible with the limit of computing load. $T_{c \max }$ is used to judge the conductor overheating and $P_{\max }$ represents tolerance of conductor overheating in the electricity sector. Both of them should be selected according to the actual requirement because the safety standards for transmission line operation are different in each country.

\section{Validation and Analysis}

\subsection{Experiment setting}

An experimental platform shown in Fig. 1 and Fig. 2 was set up on the laboratory building roof at the $20 \mathrm{~m}$ height above the ground to validate DTR estimation method. Five same aluminum-conductors steel-reinforced (ACSR), numbered 1 to 5, were adopted as the experi-

Table 1. Parameters of experimental conductor

\begin{tabular}{c|c}
\hline Parameter & Value \\
\hline Type & ACSR $120 / 25$ \\
\hline Diameter & $15.74 \mathrm{~mm}$ \\
\hline Weight & $526.6 \mathrm{~kg} \cdot \mathrm{km}^{-1}$ \\
\hline DC resistance & $0.2345 \Omega \cdot \mathrm{km}^{-1}$ \\
\hline Specific heat capacity (steel/ aluminum) & $450 / 900 \mathrm{~J} \cdot(\mathrm{kg} \cdot \mathrm{K})^{-1}$ \\
\hline
\end{tabular}


mental subjects and their specifications were shown in Table 1 . The length of every conductor was $6 \mathrm{~m}$, which was limited by the space of experimental site. Both ends of each conductor were connected tightly to form the oneturn coil. Then the five current generators, shown in the dashed box of fig.1, loaded different current levels to the corresponding conductors.

The temperature and current of each conductor were measured by a thermistor and a current transformer (CT) respectively. The thermistors were calibrated by a high precision mercurial thermometer before they were attached on the surface of conductors. The weather conditions during the experiment were measured by a weather station. All measurements was acquired by a data acquisition system with the 1-minute sampling period and then sent to a computer for the DTR estimation.

In this experimental platform, the conductor is relatively short and both ends of each conductor were connected tightly to form the one-turn coil. But in the practical engineering, the conductor is longer, straighter and therefore under more tension. Although the conductor in

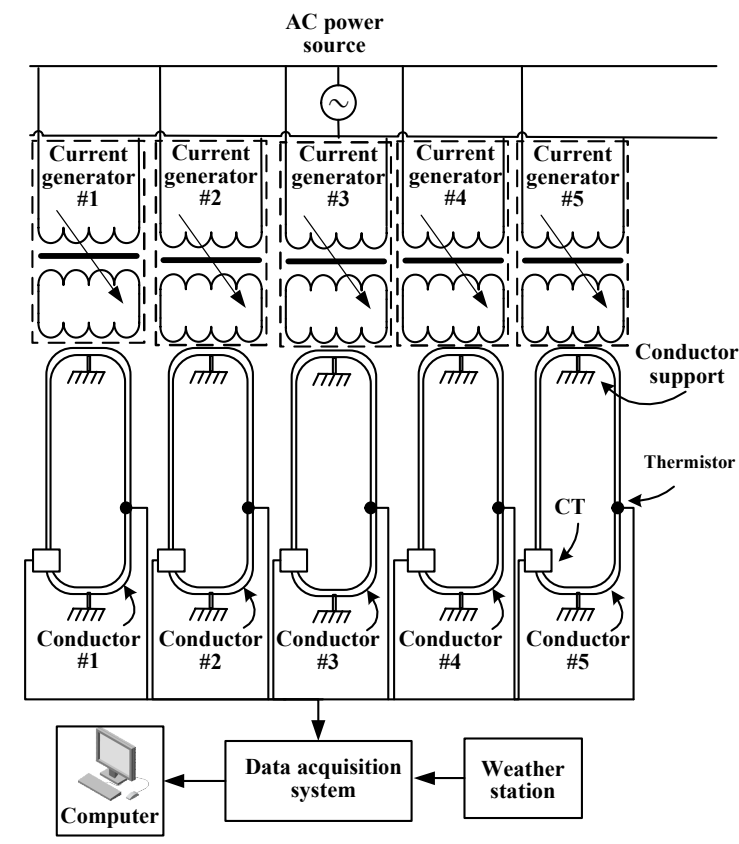

Fig. 1. Architecture of experimental platform

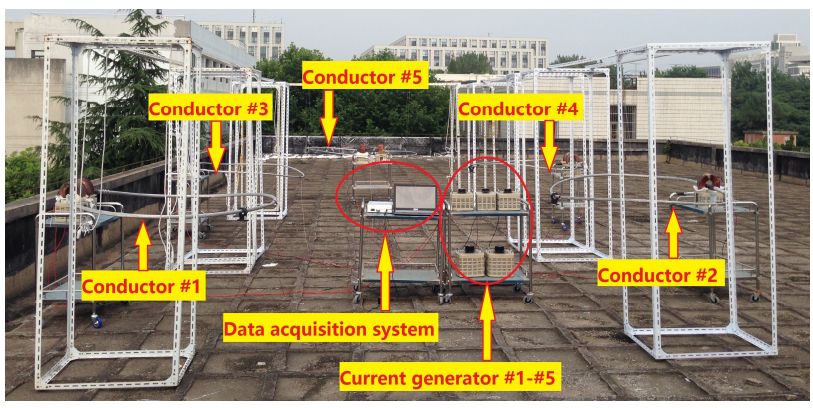

Fig. 2. Photo of experimental platform the experiment is different form the practical engineering, this experimental platform is suited to validate the proposed DTR estimation method, for the following reasons.

Firstly, the proposed method estimated DTR with the conductor temperature rather than the conductor tension. In other words, the tension is not a required parameter in the DTR estimated method and therefore the experimental platform can be set up without considering of conductor tension.

Secondly, the wind speed and its direction affect the temperature of outdoor conductor at any moment, whatever the conductor is straight or forms a coil. That means the IETR can be simulated and DTR can be estimated by the conductor temperature measured from this experimental platform.

Thirdly, the advantage of this experimental platform is that it needs less occupied space, so that the many platforms can be performed in a limited space. It makes that the conductor temperature can be observed under the same weather conditions and different current levels. This is beneficial for getting the real DTR and validating the proposed estimated method.

\subsection{Validation of proposed DTR estimation method}

In this section, an experiment, called experiment $\mathrm{A}$, were performed to validate the proposed DTR estimation method. According to the computing power of data acquisition system, the parameters mentioned in section 4 were valued as shown in Table 2. These parameters are applicable in validation and analysis of the next section too.

This experiments were carried out for $50 \mathrm{~min}$ (from 6:10 am to 7:00 am) by loading the conductors with the different current levels shown in Table 3. In this experiments, the DTR was estimated with the measured temperature of conductor $\# 1$, and the measured temperature of other conductors was used for validation. Because the length $N$ in the proposed estimation method was set to 50 , only one DTR value can be estimated in the first experiment. This is

Table 2. Parameters in the DTR estimation method

\begin{tabular}{c|c}
\hline Parameter & Value \\
\hline$N$ & 50 \\
\hline$M$ & 5000 \\
\hline$L$ & 20 \\
\hline$T_{c \max }$ & $70{ }^{\circ} \mathrm{C}$ \\
\hline$P_{\max }$ & $0.05 \%$ \\
\hline
\end{tabular}

Table 3. The current levels loaded to the conductors in the experiment

\begin{tabular}{c|c}
\hline Conductor number & Current level (A) \\
\hline$\# 1$ & 300 \\
\hline$\# 2$ & 350 \\
\hline$\# 3$ & 400 \\
\hline$\# 4$ & 450 \\
\hline$\# 5$ & 500 \\
\hline
\end{tabular}


convenient for showing the state transition probability of IETR and the overheating probability of conductor when the DTR is being estimated.

Fig. 3 and Fig. 4 shows the measured temperature of conductor \#1 and ambient weather during the experiment, respectively. It can be found that the increasing rate of temperature conductor is fast at the beginning, and slows down with the passage of time. Due to the changing of weather conditions, the conductor temperature was fluctuate during the whole experiment. Among the weather variables, the solar radiation changes slowly while the wind speed and wind direction fluctuated remarkably, just as mentioned in section 3. Because of this, the historical IETR calculated with these measurements is also fluctuate, as shown in

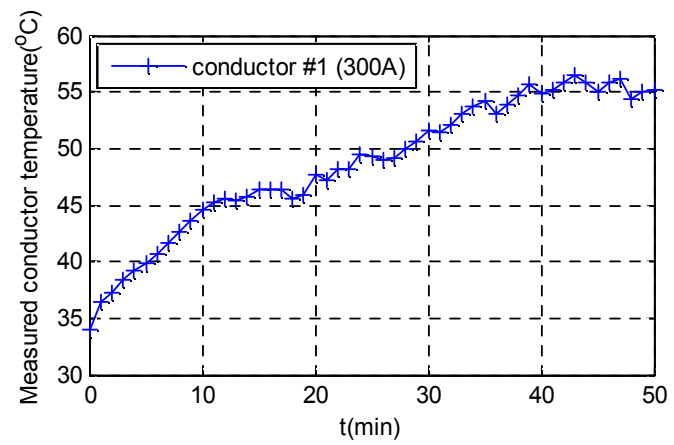

Fig. 3. The measured temperature of conductor \#1

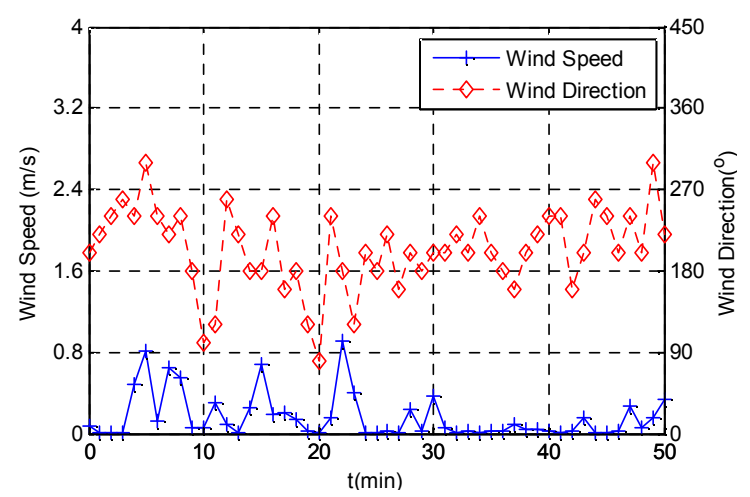

(a) Wind speed and direction

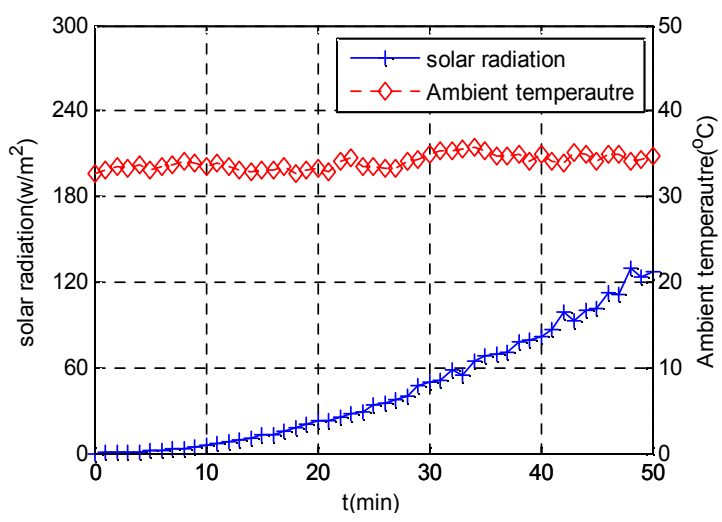

(b) Solar radiation and ambient temperature

Fig. 4. The ambient weather during the experiment A
Fig. 5 .

Fig. 6(a), (b) and (c) show, respectively, the state transition probability of historical IETR, the state transition probability of simulated IETR and the relative difference between them. It can be found that the maximum deviation between state transition probability of simulated IETR and historical IETR is only $6.3 \%$. That means the random characteristic of the historical and simulated IETR are

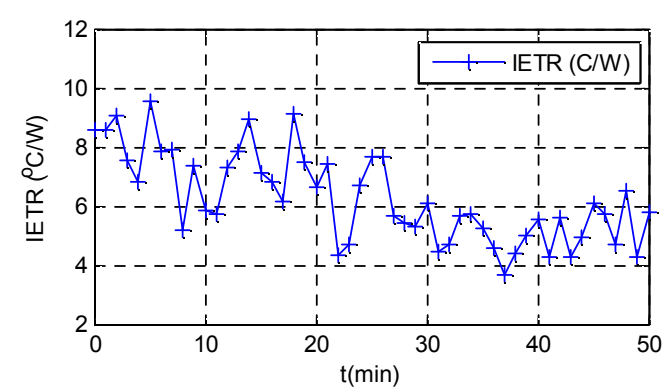

Fig. 5. The historical IETR in the experiment A

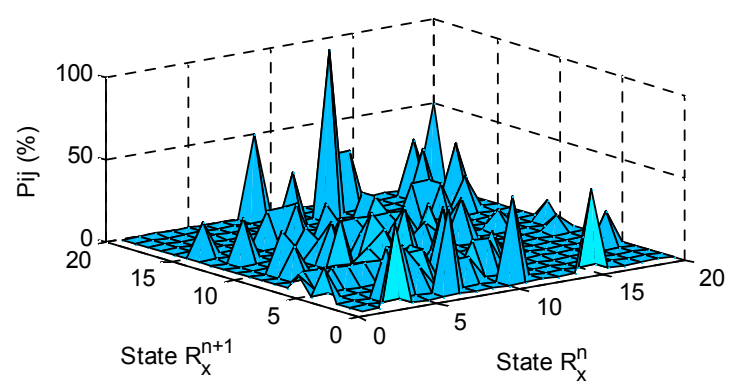

(a) The state transition probability of historical IETR

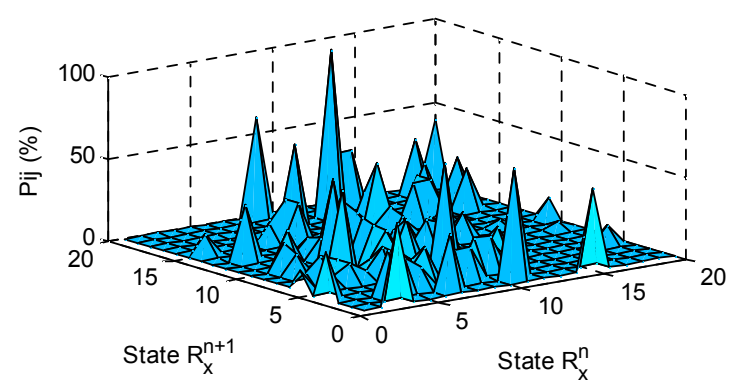

(b) The state transition probability of simulated IETR

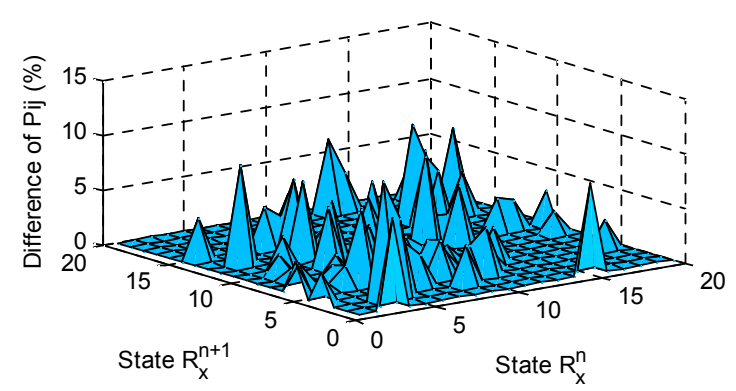

(c) The relative difference between the state transition probability of historical and simulated IETR

Fig. 6. Comparison between the state transition probability of historical and simulated IETR 


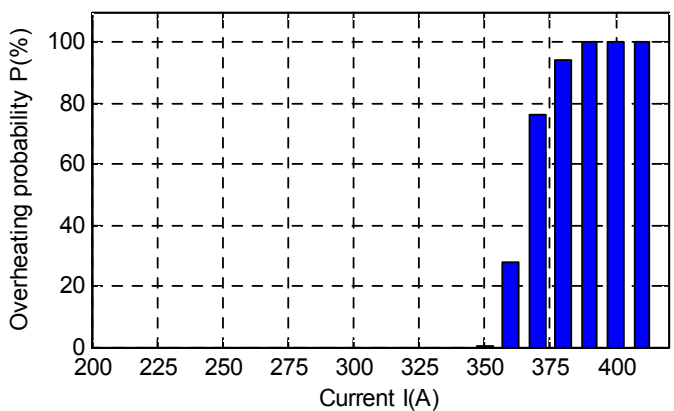

Fig. 7. The conductor overheating probability under the different current levels

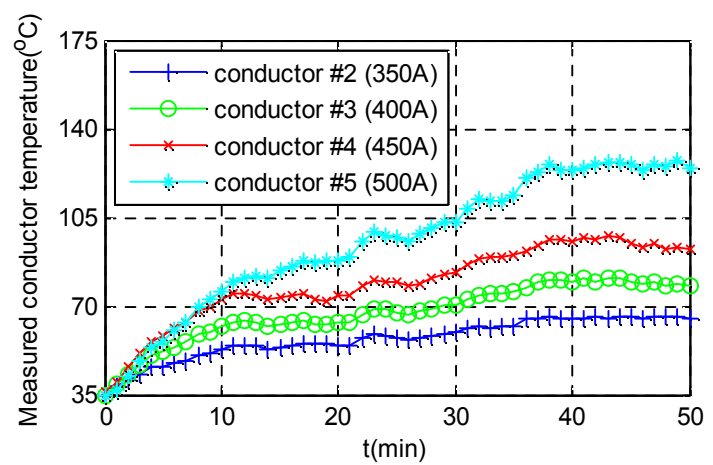

Fig. 8. The measured temperature of conductor $\# 2$ to $\# 5$

similar. It also indicate that the time series of simulated IETR, generated by Markov chain of historical IETR, can reflect the random effects of wind speed and wind direction on the conductor temperature.

Fig. 7 shows the conductor overheating probability under the different current levels, which was calculated with the proposed thermal model. The DTR at present weather conditions can be estimated as $350 \mathrm{~A}$, because it is the maximum current level whose corresponding overheating probability is less than $P_{\max }$.

The temperature of conductor \#2 to \#5 were measured to the valid DTR estimation, as shown in Fig. 8. It can be found that the conductor temperature was close to the maximum allowable value $\left(70^{\circ} \mathrm{C}\right)$ but never exceeded when the conductor was operated at 350A. Whereas, the conductor was overheated when the current levels were greater than $350 \mathrm{~A}$. That means the real DTR of conductor under the current weather conditions is about 350A, which is equal to the estimated DTR. This indicates the proposed method is effective in DTR estimation.

\subsection{Validation of the advantages of Proposed DTR estimation method}

To demonstrate the advantage of estimating DTR in the form of probability, an experiment, called experiment B, was carried out for $50 \mathrm{~min}$ by loading the conductors with the current levels shown in Table 3. Fig. 9 shows the weather conditions during this experiment and Fig. 10 shows

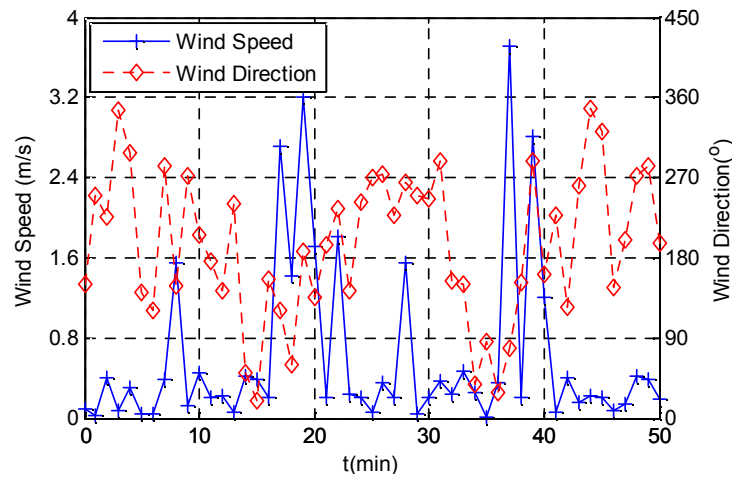

(a) Wind speed and direction

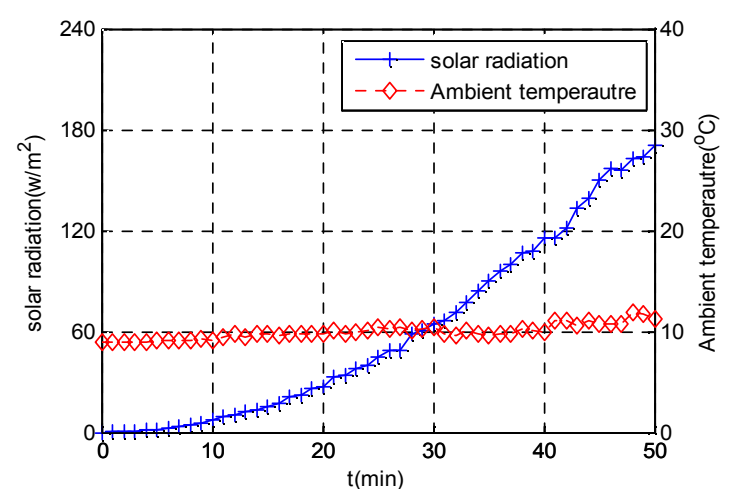

(b) Solar radiation and ambient temperature

Fig. 9. The ambient weather during the experiment B

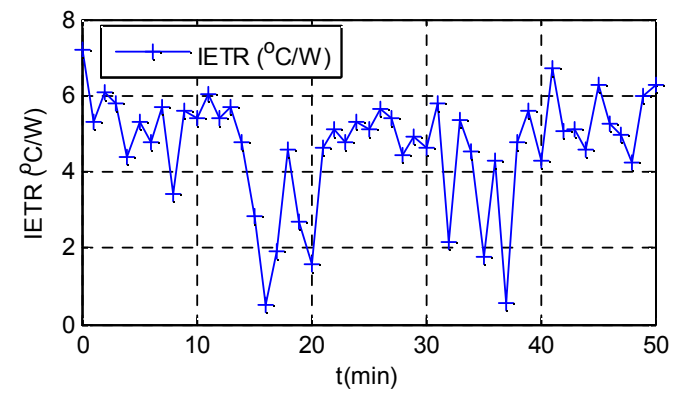

Fig. 10. The historical IETR in the experiment B

the historical IETR calculated with the measurements. It can be found that the wind speed in this experiment is increased sharply at particular time points. Since the IETR is affected by the wind speed, some values of historical IETR is changed sharply too.

Fig. 11 shows the conductor temperature measured in the experiment B. The measured temperature indicates that the real DTR in this experiment should be between $400 \mathrm{~A}$ and $450 \mathrm{~A}$ when the maximum allowable conductor temperature is set to $70^{\circ} \mathrm{C}$.

If the DTR is not estimated with the probabilistic method, the historical IETR has to be averaged to make sure that the simulated conductor temperature and the estimated DTR are deterministic. In this experiment, the averaged historical IETR is $4.13^{\circ} \mathrm{C} / \mathrm{W}$. Fig. 12 shows the 


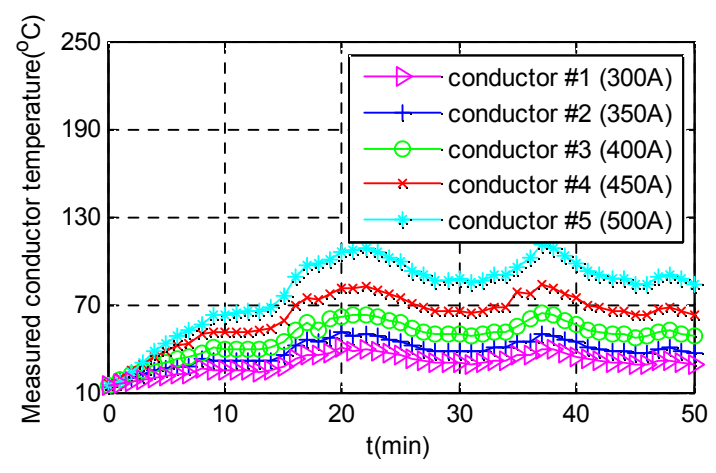

Fig. 11. The conductor temperature measured in the experiment B

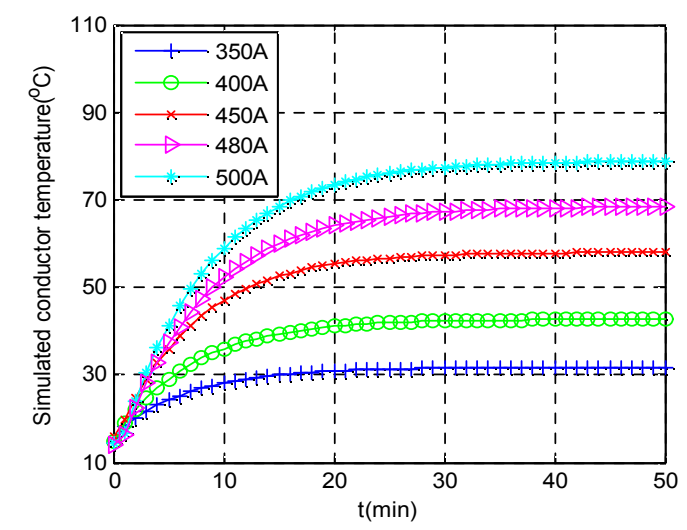

Fig. 12. The conductor temperature simulated with the average historical IETR and different current levels

conductor temperature simulated with this average historical IETR and different current levels. It can be found that there is no obvious fluctuation exist in the simulated temperature when the random characteristic of IETR is neglected. According to the simulated temperature, the DTR is estimated to be about $480 \mathrm{~A}$, which is obviously larger than real DTR. This is because the values of historical IETR, which decrease sharply at particular time points, reduce the average of historical IETR and overestimate the heat dissipation capacity of conductor. As a result, the DTR is also overestimated when the conductor temperature is calculated with the small averaged historical IETR, and this will cause the operation risk of transmission line.

Fig. 13 shows the conductor overheating probability calculated by using the proposed DTR estimated method. According to these overheating probabilities, the DTR is estimated to be 420A, which is more reliable than DTR estimated by the average historical IETR. This is because the proposed method consider the effect of random IETR on the conductor temperature.

To demonstrate the advantage of proposed DTR estimation method compared with the traditional thermal model, an experiment, called experiment $\mathrm{C}$, was preformed for 6 hours by loading the conductors \#1 with the different

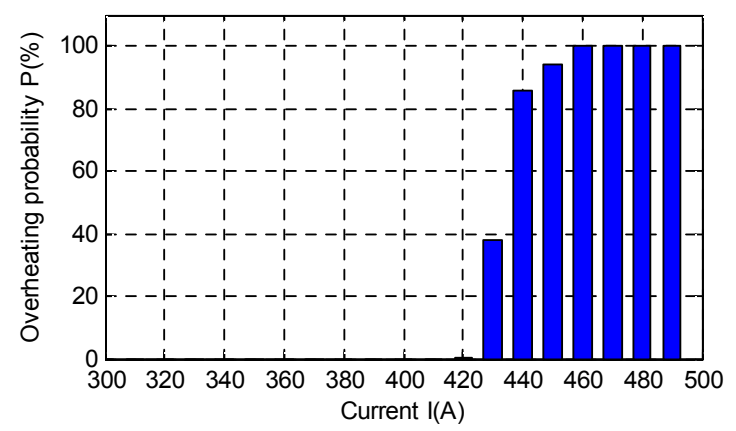

Fig. 13. The conductor overheating probability calculated by using the proposed DTR estimated method

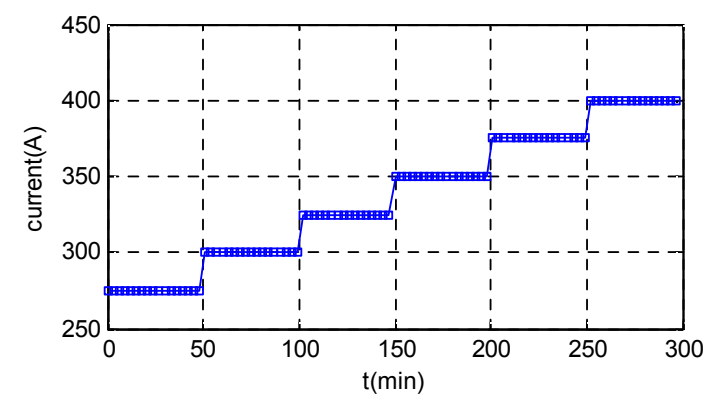

Fig. 14. The current loaded to the conductors \#1 in the experiment $\mathrm{C}$

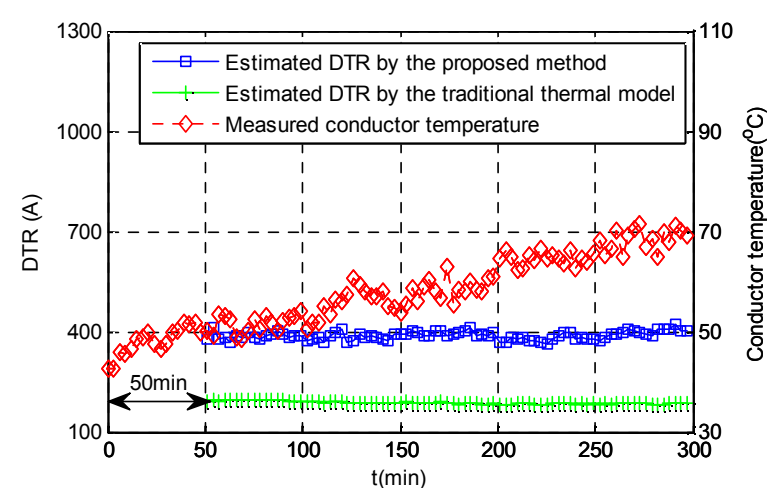

Fig. 15. The measured temperature of conductor $\# 1$ and the results of DTR estimation

current levels shown in Fig. 14.

In this experiment, the measured solar and wind data were set to zero to highlight the advantage of proposed method. In another words, the solar and wind sensors were assumed to be damaged or uninstalled.

The DTR estimated by the proposed method and traditional thermal model are illustrated in Fig. 15. Since the length $N$ in the proposed estimation method was set to 50 , the DTR in this experiment can be estimated in every minute after the 50th minute. The measured conductor temperature is also shown in Fig. 15 for obtaining the real DTR.

The data form Fig. 14 and Fig. 15 indicates that the real DTR in the experiment $\mathrm{C}$ is approximately 400A, because 
the conductor temperature just reached the maximum allowable value at the current of 400A. Due to the hypothetic failure of sensors, the heat dissipation capability of conductor is underestimated by the traditional thermal model. Consequently, the DTR determined by the traditional thermal model is approximately $180 \mathrm{~A}$ that is much smaller than real DTR value. Conversely, since the proposed DTR estimated method does not rely on the measured solar and wind data, the DTR estimated by the proposed method fluctuates slightly around 400A. This demonstrates that the proposed method is more reliable than traditional thermal model when weather sensors are out of function or uninstalled.

Furthermore, the current level was changing in the experiment C. It proves that the proposed method is effective even though the current changes dynamically and therefore is applicable to be used in the actual engineering.

\section{Conclusion}

In this study, a novel thermal model of overhead conductor is proposed based on the thermal-electric analogy theory and Markov chain. In this model, the solar, wind speed and wind direction data are no longer as the essential inputs to describe the thermal behavior of overhead conductor. By random simulation of IETR, the proposed model can be used to simulate the variations of conductor temperature with any specific current level and ambient temperature.

With the proposed thermal model, a new estimation method was presented to determine the DTR without measured wind and solar radiation. Experimental results demonstrate that the DTR estimated by the proposed method is more reliable than the traditional thermal model when the weather sensors are out of function or uninstalled. This has significance for improving the safety of overhead transmission lines when the transmission capacity need to be increased. Besides that, it is conducive to simplify DTR system structure and reduce its cost.

\section{Acknowledgements}

This work was supported by the "National Natural Science Foundation of China", no. 51607091, and "Fundamental Research Funds for the Central Universities", no. 30916011334.

\section{References}

[1] W. Q. Sun, Y. Zhang, C. M. Wang, P. Song. "Flexible load shedding strategy considering real-time dynamic thermal line rating". IET Gener. Transm. Distrib. vol. 7, no. 2, pp. 130-137, 2013.
[2] S. D. Kim, and M. M. Morcos, "An application of dynamic thermal line rating control system to up-rate the ampacity of overhead transmission lines". IEEE Transactions on Power Delivery, vol. 28, pp.12311232, Apr. 2013.

[3] H. Banakar, N. Alguacil, and F. D galiana. "Electrothermal coordination part I: theory and implementation schemes". IEEE Transactions on Power Systems, vol. 20, no. 2, pp. 798-805, May. 2005.

[4] P. Pytlak, P. Musilek, E. Lozowski, J. Toth, Modelling precipitation cooling of overhead conductors. Electric Power System Research. vol.81, pp. 2147-2154, 2011.

[5] Marcelo M, Doris S, Mark F, Identification of Critical Spans for Monitoring Systems in Dynamic Thermal Rating. IEEE Transactions on Power Delivery, vol. 27, no. 2, pp. 1002-1009, Apr. 2012

[6] T. O. Swppa, S. Damsgaard-Mikkelsen, M. Clements, R. Payne, and N. Coad, "Application of real time thermal ratings for optimizing transmission line investment and operating decisions", presented at the CIGRE, Paris, France, 2000.

[7] C. Nascimento, J. Brito, E. Filho, G, Braga, G. Miranda, A. Bracarense, and S. Ueda, "The state of the art for increased overhead line ampacity utilizing new technologies and statistical criteria", in Proc. IEEE/Power Eng. Soc. Transm. Distrib, Conf, Expo. Latin America, pp. 464-464, 2004.

[8] IEEE standard for calculating the current-temperature relationship of bare overhead conductors, IEEE Standard 738-2006, Jan. 2007.

[9] Juliano S.A. Carneiro, L. Ferrarini, "A probabilistic protection against thermal overloads of transmission lines". Electric Power Systems Research, vol. 81, pp. 1874-1880, 2011.

[10] D. M. Kim, Y. H. Park, I. S. Bae, J. O. Kim. "Decision of transmission line rating based on assessment of thermal overload risk". In Proc. The Eleventh International Middle East Power Systems Conference, pp.366-370, 2006.

[11] H. Shaker, M. F. Firuzabad, F. Aminifar. "Fuzzy dynamic thermal rating of transmission lines". IEEE Transactions on Power Delivery. vol.27, pp. 18851982, OCT, 2012.

[12] A. Piccolo, A. Vaccaro, D. Villacci, "Thermal rating assessment of overhead lines by Affine Arithmetic". Electric Power Systems Research, vol.71, pp. 275 283, 2004.

[13] R. Bernini, A. Minardo, G.V. Persiano, A. Vaccaro, D. Villacci and L. Zeni. "Dynamic loading of overhead lines by adaptive learning techniques and distributed temperature sensing”. IET Gener. Transm. Distrib., vol.1, pp. 912-919, 2007.

[14] J. Hosek, P. Musilek, E. Lozowski, P. Pytlak. "Effect of time resolution of meteorological inputs on dynamic thermal rating calculations". IET Gener. Transm. Distrib., vol. 5, pp. 941-947, 2011. 
[15] M. K. Lee, S. H. Moon, Y. H. Kim, B. R. Moon, "Correcting abnormalities in meteorological data by machine learning", 2014 IEEE International Conference on Systems, Man, and Cybernetics October 5-8, San Diego, CA, USA, 2014.

[16] A. Ammous, S. Ghedira, B. Allard, H. Morel, and D. Renault. "Choosing a thermal model for electrothermal simulation of power semiconductor devices". IEEE Trans. Power Electr., vol. 14, pp. 300-307, Mar, 1999.

[17] G. Swift, L. Senior, T. S. Molinski and W. Lehn, “A fundamental approach to transformer thermal modeling-part I: theory and equivalent circuit". IEEE Trans. Power Del. vol.16, pp.171-175, Apr. 2001.

[18] W. Z. Black, R. L. Rehberg. "Simplified model for steady state and real-time ampacity of overhead conductors". IEEE Transactions on Power Apparatus and Systems, vol. PAS-104, pp. 2942-2953, Oct. 1985

[19] H. Banakar, N. Alguacil, and F. D. Galiana. "Electrothermal Coordination Part I: Theory and Implementation Schemes". IEEE Trans. Power Syst., vol. 20, pp. 798-805, May. 2005.

[20] T. O. Seppa, S. D. Mikkelsen, M. Clements, R. Payne, and N. Coad, "Application of real time thermal ratings for optimizing transmission line investment and operating decisions", presented at the CIGRE, Paris, France, 2000.

[21] J. K. Raniga and R. K. Rayudu, "Dynamic rating of transmission lines-a New Zealand experience," in Proc. IEEE Power Eng. Soc. Winter Meeting, vol. 4, pp. 2403-2409, 2000.

[22] T. O. Seppa, "Increasing transmission capacity by real time monitoring," in Proc. IEEE Power Eng. Soc. Winter Meeting, vol. 2, pp. 1208-1211, 2002.

[23] D. U. J. Sonnadara, D. R. Jayewardene. "A Markov chain probability model to describe wet and dry patterns of weather at Colombo". Theoretical and Applied Climatology. vol. 119, pp. 333-340. Jan. 2015.

[24] H. X. Yang, Y. T. Li, L. Lu, R. Qi. "First order multivariate Markov chain model for generating annual weather data for Hong Kong". Energy and Buildings, vol.43, pp. 2371-2377, 2011.

[25] O. Kwon, Y. J. Yoon, S. K. Moon, H. J. Choi, J. H. Shim. "Estimation of Singapore's hourly solar radiation using hybrid-Markov transition matrices method", International Journal of Precision Engineering and Manufacturing. vol. 14, pp. 323-327. Feb. 2013

[26] L. L. Wilson, D. P. Lettenmaier, E. F. Wood. "Simulation of daily precipitation in the Pacific Northwest using a weather classification scheme". Surveys in Geophysics. vol. 12, pp. 127-142. 1991.

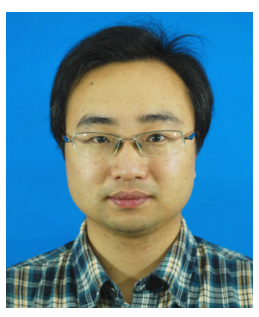

Zhan-Feng Ying He received B.S. degree in Automation Engineering from Shenyang Aerospace University, Shenyang, China, in 2004 and his M.S. degree from Nanjing University of Science and Technology, Nanjing, China in 2006. He also received the $\mathrm{Ph} . \mathrm{D}$. degree from Nanjing University of Science and Technology, in 2009. He worked as a postdoc in Electrical Engineering at Nanjing University of Science and Technology in 2011. Currently, he is an associate professor in the School of Energy and Power Engineering at Nanjing University of Science and Technology. His research interests include power quality, fault diagnosis of power transmission network and thermal management of power electronic components.

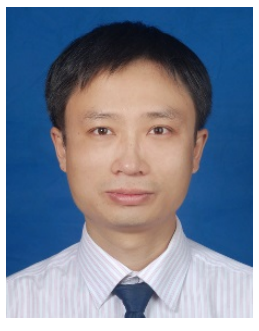

Yuan-Sheng Chen He received his B.S. degree in navigation guidance and control technology in 2005, and his $\mathrm{Ph} . \mathrm{D}$. degree in intelligent monitoring and control from Nanjing University of Aeronautics and Astronautics, Nanjing, China, in 2013. He has been a visiting scholar at Pennsylvania State University, USA, in 2010. Currently, he is a Lecturer in the School of Energy and Power Engineering at Nanjing University of Science and Technology. His research interests include intelligent monitoring of power system, piezoelectric energy harvesters, and nonlinear control of piezoelectric actuators.

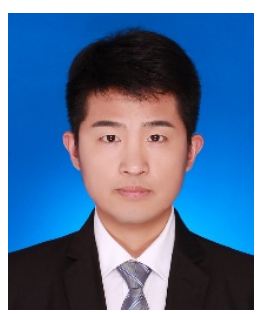

Kai Feng He received B.S. degree and M.S. degree in Electrical Engineering from Nanjing University of Science and Technology, Nanjing, China, in 2007 and 2010, respectively. He is currently pursuing his Ph.D. degree from the School of Energy and Power Engineering at Nanjing University of Science and Technology, Nanjing, China. His research interests include electro-thermal coordination theory and optimal scheduling control of power grid. 